Article

\title{
The Local Representation Formula of Solution for the Perturbed Controlled Differential Equation with Delay and Discontinuous Initial Condition
}

\author{
A. Nachaoui ${ }^{1}$, T. Shavadze ${ }^{2, *}$ and T. Tadumadze ${ }^{2,3}$ \\ 1 Jean Leray Laboratory of Mathematics, University of Nantes, CNRS UMR 66292 rue de Houssiniere, \\ B.P. 92208, 44322 Nantes, France; nachaoui@math.cnrs.fr \\ 2 I. Vekua Institute of Applied Mathematics, 2 University St., 0186 Tbilisi, Georgia; tamaz.tadumadze@tsu.ge \\ 3 Department of Mathematics, Iv. Javakhishvili Tbilisi State University, 0186 Tbilisi, Georgia \\ * Correspondence: tea.shavadze@gmail.com
}

Received: 26 August 2020; Accepted: 13 October 2020; Published: 20 October 2020

\begin{abstract}
For the perturbed controlled nonlinear delay differential equation with the discontinuous initial condition, a formula of the analytic representation of solution is proved in the left neighborhood of the endpoint of the main interval. In the formula, the effects of perturbations of the delay parameter, the initial vector, the initial and control functions are detected.
\end{abstract}

Keywords: delay-controlled differential equation; representation of solution; discontinuous initial condition; perturbations

AMS Subject Classification (2010): 34K07; 34K27

\section{Introduction}

The controlled differential equation with delay (briefly, delay-controlled equation) is a mathematical model of such a controlled dynamical system whose behavior depends on the prehistory of the system state at a given moment of time. Such mathematical models arise in different areas of natural sciences and economics [1-4]. To illustrate this, below, we will consider the simplest model of economic growth. Let $p(t)$ be a quantity of a product produced at the moment $t$, expressed in money units. The fundamental principle of the economic growth has the form

$$
p(t)=a(t)+i(t),
$$

where $a(t)$ is a quantity of money for various requirements and $i(t)$ is a quantity-induced investment. We consider the case where the functions $a(t)$ and $i(t)$ have the form

$$
a(t)=u_{1}(t) p(t)
$$

and

$$
i(t)=u_{2}(t) p(t-\tau)+\alpha \dot{p}(t),
$$

where $u_{i}(t) \in(0,1), i=1,2$, are control functions, $\alpha>0$ is a given number and $\tau>0$ is the so-called delay parameter. The Formula (3) shows that the value of investment at the moment $t$ depends on the quantity of money at the moment $t-\tau$ (in the past) and on the velocity (production current) at the moment $t$. From Formulas (1)-(3), we get the delay-controlled equation

$$
\dot{p}(t)=\frac{1-u_{1}(t)}{\alpha} p(t)-\frac{u_{2}(t)}{\alpha} p(t-\tau) .
$$


In the present paper, an analytic relation in the left neighborhood of the moment $t_{1}$ is found between the solution of the original Cauchy problem

$$
\begin{gathered}
\dot{x}(t)=f\left(t, x(t), x\left(t-\tau_{0}\right), u_{0}(t)\right), \quad t \in\left[t_{0}, t_{1}\right], \quad x(t) \in \mathbb{R}^{n}, \\
x(t)=\varphi_{0}(t), \quad t \in\left[t_{0}-\tau_{0}, t_{0}\right), \quad x\left(t_{0}\right)=x_{00}
\end{gathered}
$$

and the solution of the corresponding perturbed (with respect to delay $\tau_{0}$, initial vector $x_{00}$, initial function $\varphi_{0}(t)$ and control function $\left.u_{0}(t)\right)$ problem. The condition (5) is called the discontinuous initial condition because, in general, $x\left(t_{0}\right) \neq \varphi_{0}\left(t_{0}\right)$. Discontinuity at the initial moment may be related to the instant change in the dynamical process (changes in investment, environment, etc.). We note that such an analytic relation plays an important role in proving the necessary conditions of optimality [4-9]. Besides, such a relation allows one to analytically obtain an approximate solution of the perturbed equation in the left neighborhood of the endpoint of the main interval (see (17)).

The local representation formulas of solutions for the perturbed equations with the discontinuous initial condition for various classes of variations in terms of a parameter $\varepsilon>0$ are given: in [10] for a controlled equation; in $[7,9]$, for an equation without control. In this paper, the main novelty is the effects of the discontinuous initial condition (5) and delay perturbation detected in the local representation formula of a solution (see (9)). We note that, in this paper, the main formula is proved without a parameter $\varepsilon>0$. The formula obtained here is proved by the scheme given in [7,9], and it is essentially different from with the formula provided in [11] for the Equation (4) with the initial condition

$$
x(t)=\varphi_{0}(t), \quad t \in\left[t_{0}-\tau_{0}, t_{0}\right] .
$$

Condition (6) is called the continuous initial condition because $x\left(t_{0}\right)=\varphi_{0}\left(t_{0}\right)$ is always true.

The paper is organized as follows. In Section 2, the main theorem on the representation of solution of the perturbed equation is formulated; the effects of the discontinuous initial condition and perturbation of the initial date are analyzed; the form of equation in variations is detected; the mentioned equation is concretized for the economic growth model; two ways finding an approximate solution are given. The method developed here, based on an estimation of the increment of the solution (see Section 3) and on Cauchy's formula of the integral representation of the solutions of the linear equations. The main Theorem is proved in Section 4. Section 5 deals with the conclusion.

\section{Formulation of the Main Result}

Let $I=\left[t_{0}, t_{1}\right]$ be a finite interval and let $0<\tau_{1}<\tau_{2}$ be given numbers; suppose that $O \subset \mathbb{R}^{n}$ and $U \subset \mathbb{R}^{r}$ are open sets. Let the $n$-dimensional function $f(t, x, y, u)$ be continuous on $I \times O^{2} \times U$ and continuously differentiable with respect to $x, y$ and $u$. Let $\Phi$ be a set of continuously differentiable initial functions $\varphi: I_{1}=\left[\hat{\tau}, t_{1}\right] \rightarrow O$, where $\hat{\tau}=t_{0}-\tau_{2}$, and let $\Omega$ be a set of measurable control functions $u(t), t \in I$ satisfying the conditions: the set $\operatorname{cl} u(I) \subset U$ and is compact in $\mathbb{R}^{r}$.

To each element $\mu=\left(\tau, x_{0}, \varphi, u\right) \in \Lambda=\left[\tau_{1}, \tau_{2}\right] \times O \times \Phi \times \Omega$, we assign the delay-controlled equation

$$
\dot{x}(t)=f(t, x(t), x(t-\tau), u(t)), \quad t \in I
$$

with the discontinuous initial condition

$$
x(t)=\varphi(t), \quad t \in\left[\hat{\tau}, t_{0}\right), \quad x\left(t_{0}\right)=x_{0} .
$$

Definition 1. Let $\mu=\left(\tau, x_{0}, \varphi, u\right) \in \Lambda$. A function $x(t)=x(t ; \mu) \in O, t \in I_{1}$, is called the solution of Equation (7) with the initial condition (8) or a solution corresponding to the element $\mu$ and defined on the interval $I_{1}$, if $x(t)$ satisfies condition (8) and is absolutely continuous on the interval I, and it satisfies Equation (7) almost everywhere (a. e.) on I. 
It is clear that the solution $x(t)=x(t ; \mu), t \in I_{1}$, in general, at the point $t_{0}$, is discontinuous. Let us introduce notations

$$
|\mu|=|\tau|+\left|x_{0}\right|+\|\varphi\|_{1}+\|u\|, \quad \Lambda_{\varepsilon}\left(\mu_{0}\right)=\left\{\mu \in \Lambda:\left|\mu-\mu_{0}\right| \leq \varepsilon\right\}
$$

where

$$
\|\varphi\|_{1}=\sup \left\{|\varphi(t)|+|\dot{\varphi}(t)|: t \in I_{1}\right\}, \quad\|u\|=\sup \{|u(t)|: t \in I\},
$$

$\varepsilon>0$ is a fixed number and $\mu_{0}=\left(\tau_{0}, x_{00}, \varphi_{0}, u_{0}\right) \in \Lambda$ is a fixed element;

furthermore,

$$
\begin{gathered}
\delta \tau=\tau-\tau_{0}, \quad \delta x_{0}=x_{0}-x_{00}, \quad \delta \varphi(t)=\varphi(t)-\varphi_{0}(t), \quad \delta u(t)=u(t)-u_{0}(t), \\
\delta \mu=\mu-\mu_{0}=\left(\delta \tau, \delta x_{0}, \delta \varphi, \delta u\right), \quad|\delta \mu|=|\delta \tau|+\left|\delta x_{0}\right|+\|\delta \varphi\|_{1}+\|\delta u\| .
\end{gathered}
$$

Let $x\left(t ; \mu_{0}\right)$ be the solution corresponding with the element $\mu_{0} \in \Lambda$ and defined on the interval $I_{1}$. Then, there exists a number $\varepsilon_{1}>0$ such that, to each element $\mu \in \Lambda_{\varepsilon_{1}}\left(\mu_{0}\right)$ a corresponding solution $x(t ; \mu)$ is defined on the interval $I_{1}$ (see Lemma 1 in Section 3 ).

Theorem 1. Let $x_{0}(t):=x\left(t ; \mu_{0}\right)$ be the solution corresponding to the element $\mu_{0}=\left(\tau_{0}, x_{00}, \varphi_{0}, u_{0}\right) \in \Lambda$ and defined on the interval $I_{1}$, with $t_{0}+\tau_{0}<t_{1}$. Let $\delta>0$ and $\varepsilon_{2} \in\left(0, \varepsilon_{1}\right)$ be numbers, such that $t_{0}+\tau_{0}+\varepsilon_{2}<$ $t_{1}-\delta$. Let the function $u_{0}(t)$ be continuous at the point $t_{0}+\tau_{0}$. Then, for arbitrary $\mu \in \Lambda_{\varepsilon_{2}}\left(\mu_{0}\right)$ on the interval $\left[t_{1}-\delta, t_{1}\right]$, the following representation holds

$$
\begin{gathered}
x(t ; \mu)=x_{0}(t)+\delta x(t ; \delta \mu)+o(t ; \delta \mu) \\
\delta x(t ; \delta \mu)=Y\left(t_{0} ; t\right) \delta x_{0}+\int_{t_{0}-\tau_{0}}^{t_{0}} Y\left(s+\tau_{0} ; t\right) f_{y}\left[s+\tau_{0}\right] \delta \varphi(s) d s \\
-\left[Y\left(t_{0}+\tau_{0} ; t\right) \hat{f}+\int_{t_{0}}^{t} Y(s ; t) f_{y}[s] \dot{x}_{0}\left(s-\tau_{0}\right) d s\right] \delta \tau \\
+\int_{t_{0}}^{t} Y(s ; t) f_{u}[s] \delta u(s) d s
\end{gathered}
$$

and

$$
\lim _{|\delta \mu| \rightarrow 0}|o(t ; \delta \mu)| /|\delta \mu|=0 \text { uniformly for } t \in\left[t_{1}-\delta, t_{1}\right] \text {. }
$$

Here,

$$
\begin{gathered}
\hat{f}=f\left(t_{0}+\tau_{0}, x_{0}\left(t_{0}+\tau_{0}\right), x_{00}, u_{0}\left(t_{0}+\tau_{0}\right)\right)-f\left(t_{0}+\tau_{0}, x_{0}\left(t_{0}+\tau_{0}\right), \varphi_{0}\left(t_{0}\right), u_{0}\left(t_{0}+\tau_{0}\right)\right) . \\
f_{y}[s]=f_{y}\left(s, x_{0}(s), x_{0}\left(s-\tau_{0}\right), u_{0}(s)\right), \quad f_{u}[s]=f_{u}\left(s, x_{0}(s), x_{0}\left(s-\tau_{0}\right), u_{0}(s)\right)
\end{gathered}
$$

$Y(s ; t)$ is the $n \times n$-matrix function satisfying the equation

$$
Y_{s}(s ; t)=-Y(s ; t) f_{x}[s]-Y\left(s+\tau_{0} ; t\right) f_{y}\left[s+\tau_{0}\right], \quad s \in\left[t_{0}, t\right]
$$

and the condition

$$
Y(s ; t)=\left\{\begin{array}{l}
E, \text { for } s=t \\
\Theta, \text { for } s>t
\end{array}\right.
$$

$f_{x}[s]=f_{x}\left(s, x_{0}(s), x_{0}\left(s-\tau_{0}\right), u_{0}(s)\right), \quad E$ is the identity matrix and $\Theta$ is the zero matrix.

\section{Some Comments}


The function $\delta x(t ; \delta \mu)$ is called the first variation of the solution $x_{0}(t)$ on the interval $\left[t_{1}-\delta, t_{1}\right]$. The expression (10) is called the variation formula of a solution. The term "variation formula of solution" was introduced by R. V. Gamkrelidze and proved in [6] for ODEs without control.

The expression

$$
Y\left(t_{0} ; t\right) \delta x_{0}+\int_{t_{0}-\tau_{0}}^{t_{0}} Y\left(s+\tau_{0} ; t\right) f_{y}\left[s+\tau_{0}\right] \delta \varphi(s) d s
$$

in Formula (10) is the effect of perturbations of the initial vector $x_{00}$ and the initial function $\varphi_{0}(t)$.

The added

$$
-\left\{Y\left(t_{0}+\tau_{0} ; t\right) \hat{f}+\int_{t_{0}}^{t} Y(s ; t) f_{y}[s] \dot{x}_{0}\left(s-\tau_{0}\right) d s\right\} \delta \tau
$$

in Formula (10) is the effect of perturbation of the delay $\tau_{0}$. Here, $Y\left(t_{0}+\tau_{0} ; t\right) \hat{f}$ is the effect of the discontinuity of the initial condition (8). If we have the continuous initial condition (6), i.e., $\varphi_{0}\left(t_{0}\right)=x_{00}$ then $\hat{f}=0$.

The expression

$$
\int_{t_{0}}^{t} Y(s ; t) f_{u}[s] \delta u(s) d s
$$

in Formula (10) is the effect of perturbation of the control function $u_{0}(t)$.

It is clear that

$$
\delta x(t ; \delta \mu)=\delta x_{1}(t ; \delta \mu)+\delta x_{2}(t ; \delta \mu)
$$

where

$$
\begin{gathered}
\delta x_{1}(t ; \delta \mu)=Y\left(t_{0} ; t\right) \delta x_{0}+\int_{t_{0}-\tau_{0}}^{t_{0}} Y\left(s+\tau_{0} ; t\right) f_{y}\left[s+\tau_{0}\right] \delta \varphi(s) d s \\
-\left[\int_{t_{0}}^{t} Y(s ; t) f_{y}[s] \dot{x}_{0}\left(s-\tau_{0}\right) d s\right] \delta \tau+\int_{t_{0}}^{t} Y(s ; t) f_{u}[s] \delta u(s) d s
\end{gathered}
$$

and

$$
\delta x_{2}(t ; \delta \mu)=-Y\left(t_{0}+\tau_{0} ; t\right) \hat{f} \delta \tau .
$$

On the basis of the Cauchy Formula (see [9], p. 31) for the representation of the solutions of the linear delay equation, we conclude that the function

$$
\delta x_{1}(t)=\left\{\begin{array}{l}
\delta \varphi(t), t \in\left[\hat{\tau}, t_{0}\right) \\
\delta x_{0}, t=t_{0} \\
\delta x_{1}(t ; \delta \mu), t \in\left(t_{0}, t_{1}\right]
\end{array}\right.
$$

is a solution of the equation

$$
\begin{aligned}
\dot{\delta} x(t)=f_{x}[t] \delta x(t) & +f_{y}[t] \delta x\left(t-\tau_{0}\right)-f_{y}[t] \dot{x}_{0}\left(t-\tau_{0}\right) \delta \tau \\
& +f_{u}[t] \delta u(t), t \in I
\end{aligned}
$$

with the initial condition

$$
\delta x(t)=\delta \varphi(t), t \in\left[\hat{\tau}, t_{0}\right), \delta x\left(t_{0}\right)=\delta x_{0} .
$$

(13) is called the equation in "variations".

The function

$$
\delta x_{2}(t)=\left\{\begin{array}{l}
0, t \in\left[\hat{\tau}, t_{0}+\tau_{0}\right) \\
-\hat{f} \delta \tau, t=t_{0}+\tau_{0} \\
\delta x_{2}(t ; \delta \mu), t \in\left(t_{0}+\tau_{0}, t_{1}\right]
\end{array}\right.
$$


is a solution of the equation

$$
\dot{\delta} x(t)=f_{x}[t] \delta x(t)+f_{y}[t] \delta x\left(t-\tau_{0}\right), t \in\left(t_{0}+\tau_{0}, t_{1}\right]
$$

with the initial condition

$$
\delta x(t)=0, t \in\left[\hat{\tau}, t_{0}+\tau_{0}\right), \delta x\left(t_{0}+\tau_{0}\right)=-\hat{f} \delta \tau .
$$

For example, for the economical model (see the Introduction)

$$
\dot{p}(t)=\frac{1-u_{10}(t)}{\alpha} p(t)-\frac{u_{20}(t)}{\alpha} p\left(t-\tau_{0}\right), t \in I
$$

with the initial condition

$$
p(t)=q_{0}(t), t \in\left[\hat{\tau}, t_{0}\right), p\left(t_{0}\right)=p_{00}
$$

the corresponding equations in "variations" and the initial conditions have the form

$$
\left\{\begin{array}{l}
\dot{\delta} p(t)=\frac{1-u_{10}(t)}{\alpha} \delta p(t)-\frac{u_{20}(t)}{\alpha} \delta p\left(t-\tau_{0}\right)+\frac{u_{20}(t)}{\alpha} \dot{p}_{0}\left(t-\tau_{0}\right) \delta \tau-\frac{p_{0}(t)}{\alpha} \delta u_{1}(t) \\
-\frac{p_{0}\left(t-\tau_{0}\right)}{\alpha} \delta u_{2}(t), t \in I ; \delta p(t)=\delta q(t), t \in\left[\hat{\tau}, t_{0}\right), \delta p\left(t_{0}\right)=\delta p_{0}
\end{array}\right.
$$

and

$$
\left\{\begin{array}{l}
\dot{\delta} p(t)=\frac{1-u_{10}(t)}{\alpha} \delta p(t)-\frac{u_{20}(t)}{\alpha} \delta p\left(t-\tau_{0}\right), t \in\left(t_{0}+\tau_{0}, t_{1}\right] \\
\delta p(t)=0, t \in\left[\hat{\tau}, t_{0}+\tau_{0}\right), \delta p\left(t_{0}+\tau_{0}\right)=\frac{u_{20}\left(t_{0}+\tau_{0}\right)}{\alpha}\left(p_{00}-q_{0}\left(t_{0}\right)\right) \delta \tau .
\end{array}\right.
$$

Formula (9) allows us to obtain an approximate solution of the perturbed equation in the analytical form on the interval $\left[t_{1}-\delta, t_{1}\right]$. In fact, for a small $|\delta \mu|$ from (9), it follows

$$
x(t ; \mu) \approx x_{0}(t)+\delta x(t ; \delta \mu), t \in\left[t_{1}-\delta, t_{1}\right] .
$$

We note that $\delta x(t ; \delta \mu)$ can be calculated by two ways: first, (see (10)-(12)) find the matrix function $Y(\xi ; t)$; second, find the solutions of Equations (13) and (15) with the initial conditions (14) and (16), respectively.

\section{Auxiliary Assertions}

Lemma $1([9](\mathbf{p} .18))$. Let $x_{0}(t)=x\left(t ; \mu_{0}\right)$ be the solution corresponding to the element $\mu_{0}=$ $\left(\tau_{0}, x_{00}, \varphi_{0}, u_{0}\right) \in \Lambda$, defined on the interval $I_{1}$. Then, there is a number $\varepsilon_{1}>0$ such that, to each element $\mu=\left(\tau, x_{0}, \varphi, u\right) \in \Lambda_{\varepsilon_{1}}\left(\mu_{0}\right)$ a corresponding solution $x(t)=x(t ; \mu)$ is defined on the interval $I_{1}$ with $x(t) \in K_{1}$ and $u(t) \in U_{1}$, where $K_{1} \subset O$ is a compact set containing a neighborhood of the set $x_{0}\left(I_{1}\right)$ and $U_{1} \subset U$ is a compact set containing a neighborhood of the set $c l u_{0}(I)$.

Lemma 1 allows one to introduce the increment of the solution $x_{0}(t), \quad t \in I_{1}$

$$
\Delta x(t):=\Delta x(t ; \delta \mu):=x(t ; \mu)-x_{0}(t), \quad t \in I_{1}, \quad \mu \in \Lambda_{\varepsilon_{1}}\left(\mu_{0}\right), \quad \delta \mu=\mu-\mu_{0} .
$$

Lemma 2. For arbitrary $\mu \in \Lambda_{\varepsilon_{1}}\left(\mu_{0}\right)$ the following inequality holds

$$
\sup _{t \in I_{1}}|\Delta x(t)| \leq O(\delta \mu),
$$


where

$$
\lim _{|\delta \mu| \rightarrow 0} O(\delta \mu) /|\delta \mu|<\infty .
$$

The Lemma 2 without principle changes can be proved analogously to Lemma 2 (see [11]).

\section{Proof of Theorem 1}

Let $\varepsilon_{2} \in\left(0, \varepsilon_{1}\right)$ and $\delta>0$ be numbers such that $t_{0}+\tau_{0}+\varepsilon_{2}<t_{1}-\delta$. For $\forall \mu \in \Lambda_{\varepsilon_{2}}\left(\mu_{0}\right)=\{\mu \in$ $\left.\Lambda:\left|\mu-\mu_{0}\right| \leq \varepsilon_{2}\right\}$ we have

$$
t_{0}+\tau=t_{0}+\tau_{0}+\tau-\tau_{0} \leq t_{0}+\tau_{0}+\varepsilon_{2}<t_{1}-\delta .
$$

The function $\Delta x(t)=x(t)-x_{0}(t)$ satisfies the equation

$$
\dot{\Delta} x(t)=f_{x}[t] \Delta x(t)+f_{y}[t] \Delta x\left(t-\tau_{0}\right)+f_{u}[t] \delta u(t)+r(t ; \delta \mu)
$$

on the interval $I$ and the initial condition

$$
\Delta x(t)=\delta \varphi(t), t \in\left[\hat{\tau}, t_{0}\right), \Delta x\left(t_{0}\right)=\delta x_{0} .
$$

Here

$$
\left\{\begin{array}{l}
r(t ; \delta \mu):=a(t ; \delta \mu)-f_{x}[t] \Delta x(t)-f_{y}[t] \Delta x\left(t-\tau_{0}\right)-f_{u}[t] \delta u(t), \\
a(t ; \delta \mu)=f\left(t, x_{0}(t)+\Delta x(t), x_{0}(t-\tau)+\Delta x(t-\tau), u(t)\right)-f[t] \\
f[t]=f\left(t, x_{0}(t), x_{0}\left(t-\tau_{0}\right), u_{0}(t)\right) .
\end{array}\right.
$$

By using the Cauchy formula, one can represent the solution of Equation (20) in the form

$$
\begin{gathered}
\Delta x(t)=Y\left(t_{0} ; t\right) \delta x_{0}+\int_{t_{0}-\tau_{0}}^{t_{0}} Y\left(s+\tau_{0} ; t\right) f_{y}\left[s+\tau_{0}\right] \delta \varphi(s) d s \\
+\int_{t_{0}}^{t} Y(s ; t) f_{u}[s] \delta u(s) d s+R(t ; \delta \mu),
\end{gathered}
$$

where $Y(s ; t)$ is the $n \times n$-matrix function satisfying Equation (11) and the condition (12), and

$$
R(t ; \delta \mu)=\int_{t_{0}}^{t} Y(s ; t) r(s ; \delta \mu) d s .
$$

Let $s_{1}=\min \left\{t_{0}+\tau, t_{0}+\tau_{0}\right\}, s_{2}=\max \left\{t_{0}+\tau, t_{0}+\tau_{0}\right\}$. It is clear that $s_{2}<t_{1}-\delta$ (see (19)). Therefore, for $t \in\left[t_{1}-\delta, t_{1}\right]$ we have

$$
R(t ; \delta \mu)=\sum_{i=1}^{3} R_{i}(t ; \delta \mu)
$$

where

$$
\begin{gathered}
R_{1}(t ; \delta \mu)=\int_{t_{0}}^{s_{1}} Y(s ; t) r(s ; \delta \mu) d s, R_{2}(t ; \delta \mu)=\int_{s_{1}}^{s_{2}} Y(s ; t) r(s ; \delta \mu) d s, \\
R_{3}(t ; \delta \mu)=\int_{s_{2}}^{t} Y(s ; t) r(s ; \delta \mu) d s .
\end{gathered}
$$

We introduce the notations:

$$
\begin{gathered}
f[t ; \theta, \delta \mu]=f\left(t, x_{0}(t)+\theta \Delta x(t), x_{0}\left(t-\tau_{0}\right)+\theta\left[x_{0}(t-\tau)-x_{0}\left(t-\tau_{0}\right)+\Delta x(t-\tau)\right],\right. \\
\left.u_{0}(t)+\theta \delta u(t)\right) ; \sigma(t ; \theta, \delta \mu)=f_{x}[t ; \theta, \delta \mu]-f_{x}[t], \rho(t ; \theta, \delta \mu)=f_{y}[t ; \theta, \delta \mu]-f_{y}[t] \\
v(t ; \theta, \delta \mu)=f_{u}[t ; \theta, \delta \mu]-f_{u}[t] .
\end{gathered}
$$


It is easy to see that

$$
\begin{gathered}
a(t ; \delta \mu)=\int_{0}^{1} \frac{d}{d \theta} f[t ; \theta, \delta \mu] d \theta=\int_{0}^{1}\left\{f_{x}[t ; \theta, \delta \mu] \Delta x(t)+f_{y}[t ; \theta, \delta \mu]\left[x_{0}(t-\tau)-x_{0}\left(t-\tau_{0}\right)\right.\right. \\
\left.+\Delta x(t-\tau)]+f_{u}[t ; \theta, \delta \mu] \delta u(t)\right\} d \theta=\left[\int_{0}^{1} \sigma(t ; \theta, \delta \mu) d \theta\right] \Delta x(t)+ \\
+\left[\int_{0}^{1} \rho(t ; \theta, \delta \mu) d \theta\right]\left(x_{0}(t-\tau)-x_{0}\left(t-\tau_{0}\right)+\Delta x(t-\tau)\right)+\left[\int_{0}^{1} v(t ; \theta, \delta \mu) d \theta\right] \delta u(t) \\
+f_{x}[t] \Delta x(t)+f_{y}[t]\left[x_{0}(t-\tau)-x_{0}\left(t-\tau_{0}\right)+\Delta x(t-\tau)\right]+f_{u}[t] \delta u(t)=\sigma_{1}(t ; \delta \mu) \Delta x(t) \\
+\rho_{1}(t ; \delta \mu)\left(x_{0}(t-\tau)-x_{0}\left(t-\tau_{0}\right)+\Delta x(t-\tau)\right)+v_{1}(t ; \delta \mu) \delta u(t)+f_{x}[t] \Delta x(t) \\
+f_{y}[t]\left[x_{0}(t-\tau)-x_{0}\left(t-\tau_{0}\right)+\Delta x(t-\tau)\right]+f_{u}[t] \delta u(t),
\end{gathered}
$$

where

$$
\begin{gathered}
\sigma_{1}(t ; \delta \mu)=\int_{0}^{1} \sigma(t ; \theta, \delta \mu) d \theta, \rho_{1}(t ; \delta \mu)=\int_{0}^{1} \rho(t ; \theta, \delta \mu) d \theta \\
v_{1}(t ; \delta \mu)=\int_{0}^{1} v(t ; \theta, \delta \mu) d \theta
\end{gathered}
$$

Taking into account the last relation, we have

$$
R_{1}(t ; \delta \mu)=\sum_{i=1}^{5} R_{1 i}(t ; \delta \mu)
$$

where

$$
\begin{gathered}
R_{11}(t ; \delta \mu)=\int_{t_{0}}^{s_{1}} Y(s ; t) \sigma_{1}(s ; \delta \mu) \Delta x(s) d s \\
R_{12}(t ; \delta \mu)=\int_{t_{0}}^{s_{1}} Y(s ; t) \rho_{1}(s ; \delta \mu)\left[x_{0}(s-\tau)-x_{0}\left(s-\tau_{0}\right)+\Delta x(s-\tau)\right] d s, \\
R_{13}(t ; \delta \mu)=\int_{t_{0}}^{s_{1}} Y(s ; t) f_{y}[s]\left[x_{0}(s-\tau)-x_{0}\left(s-\tau_{0}\right)\right] d s, \\
R_{14}(t ; \delta \mu)=\int_{t_{0}}^{s_{1}} Y(s ; t) f_{y}[s]\left[\Delta x(s-\tau)-\Delta x\left(s-\tau_{0}\right)\right] d s, \\
R_{15}(t ; \delta \mu)=\int_{t_{0}}^{s_{1}} Y(s ; t) v_{1}(s ; \delta \mu) \delta u(s) d s .
\end{gathered}
$$

The function $\varphi_{0}(s), s \in I_{1}$, is continuously differentiable, therefore, for each fixed point $s \in I$, we obtain

$$
\varphi_{0}(s-\tau)-\varphi_{0}\left(s-\tau_{0}\right)=\int_{s}^{s-\delta \tau} \dot{\varphi}_{0}\left(s-\tau_{0}\right) d \varsigma=-\dot{\varphi}_{0}\left(s-\tau_{0}\right) \delta \tau+\gamma(s ; \delta \tau)
$$

where

$$
\lim _{|\delta \tau| \rightarrow 0} \gamma(s ; \delta \tau) /|\delta \tau|=0
$$

From (23) it follows that

$$
\left|\varphi_{0}(s-\tau)-\varphi_{0}\left(s-\tau_{0}\right)\right| \leq O(\delta \mu)
$$

and

$$
|\gamma(s ; \delta \tau)| /|\delta \tau| \leq \text { const } \quad \forall s \in I .
$$

For $s \in\left[t_{0}, s_{1}\right]$ we have $s-\tau<t_{0}$ and $s-\tau_{0}<t_{0}$. Therefore,

$$
\left|x_{0}(s-\tau)-x_{0}\left(s-\tau_{0}\right)\right|=\left|\varphi_{0}(s-\tau)-\varphi_{0}\left(s-\tau_{0}\right)\right| \leq O(\delta \mu)
$$


and

$$
\begin{gathered}
\left|\Delta x(s-\tau)-\Delta x\left(s-\tau_{0}\right)\right|=\left|\delta \varphi(s-\tau)-\delta \varphi\left(s-\tau_{0}\right)\right| \\
\leq\left|\int_{s-\tau_{0}}^{s-\tau}\right| \dot{\delta} \varphi(s)|d s| \leq|\delta \mu||\delta \tau|=o(\delta \mu) .
\end{gathered}
$$

According to (18), (23), (25), (26) for the expressions $R_{1 i}(t ; \delta \mu), i=1, \ldots, 5$ we have

$$
\begin{gathered}
\left|R_{11}(t ; \delta \mu)\right| \leq\|Y\| O(\delta \mu) \sigma_{2}(\delta \mu), \text { where } \quad \sigma_{2}(\delta \mu)=\int_{I}\left|\sigma_{1}(s ; \delta \mu)\right| d s ;\left|R_{12}(t ; \delta \mu)\right| \\
\left|R_{12}(t ; \delta \mu)\right| \leq\|Y\| O(\delta \mu) \rho_{2}(\delta \mu), \text { where } \quad \rho_{2}(\delta \mu)=\int_{I}\left|\rho_{1}(s ; \delta \mu)\right| d s ; R_{13}(t ; \delta \mu) \\
=-\left[\int_{t_{0}}^{s_{1}} Y(s ; t) f_{y}[s] \dot{\varphi}_{0}\left(s-\tau_{0}\right) d s\right] \delta \tau+\gamma_{1}(t ; \delta \mu) ;\left|R_{14}(t ; \delta \mu)\right| \leq o(\delta \mu), \\
\left|R_{15}(t ; \delta \mu)\right| \leq\|Y\| O(\delta \mu) v_{2}(\delta \mu), \text { where } \quad v_{2}(\delta \mu)=\int_{I}\left|v_{1}(s ; \delta \mu)\right| d s,
\end{gathered}
$$

where

$$
\|Y\|=\sup \left\{|Y(s ; t)|:(s, t) \in I^{2}\right\}, \gamma_{1}(t ; \delta \tau)=\int_{t_{0}}^{t} Y(s ; t) f_{y}[s] \gamma(s ; \delta \tau) d s
$$

Obviously,

$$
\left|\gamma_{1}(t ; \delta \tau)\right| /|\delta \mu| \leq\|Y\| \int_{I}\left|f_{y}[s]\right||\gamma(s ; \delta \tau)| /|\delta \mu| d s \leq\|Y\| \int_{I}\left|f_{y}[s]\right||\gamma(s ; \delta \tau)| /|\delta \tau| d s .
$$

By the Lebesgue theorem on the passage to the limit under the integral sign, we have

$$
\begin{gathered}
\lim _{|\delta \mu| \rightarrow 0} \sigma_{2}(\delta \mu)=0, \lim _{|\delta \mu| \rightarrow 0} \rho_{2}(\delta \mu)=0, \lim _{|\delta \mu| \rightarrow 0} v_{2}(\delta \mu)=0, \\
\lim _{|\delta \mu| \rightarrow 0}\left|\gamma_{1}(t ; \delta \mu)\right| /|\delta \mu|=0, \text { uniformly for } t \in I
\end{gathered}
$$

(see (24)). Then, for $t \in\left[t_{1}-\delta, t_{1}\right]$ we have

$$
\begin{gathered}
R_{1 i}(t, \delta \mu)=o(t ; \delta \mu), i=1,2,4,5, \\
R_{13}(t ; \delta \mu)=-\left[\int_{t_{0}}^{s_{1}} Y(s ; t) f_{y}[s] \dot{\varphi}_{0}\left(s-\tau_{0}\right) d s\right] \delta \tau+o(t ; \delta \mu) \\
=-\left[\int_{t_{0}}^{s_{1}} Y(s ; t) f_{y}[s] \dot{x}_{0}\left(s-\tau_{0}\right) d s\right] \delta \tau+o(t ; \delta \mu) .
\end{gathered}
$$

Thus,

$$
R_{1}(t ; \delta \mu)=-\left[\int_{t_{0}}^{s_{1}} Y(s ; t) f_{y}[s] \dot{x}_{0}\left(s-\tau_{0}\right) d s\right] \delta \tau+o(t ; \delta \mu)
$$

(see (27)).

Now, we transform the expression

$$
R_{2}(t ; \delta \mu)=\vartheta_{1}(t ; \delta \mu)+\vartheta_{2}(t ; \delta \mu),
$$

where

$$
\begin{gathered}
\vartheta_{1}(t ; \delta \mu)=\int_{s_{1}}^{s_{2}} Y(s ; t) a(s ; \delta \mu) d s, \vartheta_{2}(t ; \delta \mu)=-\int_{s_{1}}^{s_{2}} Y(s ; t)\left[f_{x}[s] \Delta x(s)\right. \\
\left.+f_{y}[s] \Delta x\left(s-\tau_{0}\right)+f_{u}[s] \delta u(s)\right] d s .
\end{gathered}
$$


It is clear that

$$
\left|\vartheta_{2}(t ; \delta \mu)\right| \leq O(\delta \mu)\|Y\| \int_{s_{1}}^{s_{2}}\left[\left|f_{x}[s]\right|+\left|f_{y}[s]\right|+\left|f_{u}[s]\right|\right] d s=o(\delta \mu)
$$

(see (18)). Next, let $s_{1}=t_{0}+\tau$ and $s_{2}=t_{0}+\tau_{0}$, then for $s \in\left(t_{0}+\tau, t_{0}+\tau_{0}\right)$, we have $s-\tau>t_{0}$ and $s-\tau_{0}<t_{0}$, i.e.,

$$
\begin{gathered}
a(s ; \delta \mu)=f\left(s, x_{0}(s)+\Delta x(s), x_{0}(s-\tau)+\Delta x(s-\tau), u_{0}(s)+\delta u(s)\right) \\
-f\left(s, x_{0}(s), \varphi_{0}\left(s-\tau_{0}\right), u_{0}(s)\right)
\end{gathered}
$$

(see (21)). For $|\delta \mu| \rightarrow 0$, we have $s \rightarrow t_{0}+\tau_{0}$ and $s-\tau \rightarrow t_{0}+, s-\tau_{0} \rightarrow t_{0}-$. Thus, using the continuity of the function $u_{0}(t)$ at the point $t_{0}+\tau_{0 \prime \prime}$ in this case, we obtain

$$
\lim _{|\delta \mu| \rightarrow 0} a(s ; \delta \mu)=\hat{f}
$$

It is easy to see that

$$
\vartheta_{1}(t ; \delta \mu)=-Y\left(t_{0}+\tau_{0} ; t\right) \hat{f} \delta \tau+h_{1}(t ; \delta \mu)+h_{2}(t ; \delta \mu)
$$

where

$$
\begin{gathered}
h_{1}(t ; \delta \mu)=\int_{t_{0}+\tau}^{t_{0}+\tau_{0}}\left[Y(s ; t)-Y\left(t_{0}+\tau_{0} ; t\right)\right] \hat{f} d s \\
h_{2}(t ; \delta \mu)=\int_{t_{0}+\tau}^{t_{0}+\tau_{0}} Y(s ; t)[a(s ; \delta \mu)-\hat{f}] d s .
\end{gathered}
$$

The matrix function $Y(s ; t)$ is continuous on the set $\Pi=\left\{(s, t) ; s \in\left[t_{0}, t_{0}+\tau_{0}+\varepsilon_{2}\right], t \in\left[t_{1}-\delta, t_{1}\right]\right\}$ by Lemma 2.6 (see [9], p. 32). Thus, the function $Y(s ; t)$ is uniformly continuous on $\Pi$, therefore

$$
h_{3}(t)=\sup _{s \in\left[t_{0}+\tau, t_{0}+\tau_{0}\right]}\left|Y(s ; t)-Y\left(t_{0}+\tau_{0} ; t\right)\right| \rightarrow 0 \text { for }|\delta \mu| \rightarrow 0,
$$

uniformly for $t \in\left[t_{1}-\delta, t_{1}\right]$. We have

$$
\left|h_{1}(t ; \delta \mu)\right| \leq h_{3}(t)|\delta \tau| \leq|\delta \mu| h_{3}(t)=o(t ; \delta \mu)
$$

and

$$
\left|h_{2}(t ; \delta \mu)\right| \leq\|Y\| \sup _{s \in\left[t_{0}+\tau, t_{0}+\tau_{0}\right]}|a(s ; \delta \mu)-\hat{f}||\delta \tau| \leq o(\delta \mu) .
$$

Thus, in this case

$$
R_{2}(t ; \delta \mu)=-Y\left(t_{0}+\tau_{0} ; t\right) \hat{f} \delta \tau+o(t ; \delta \mu) .
$$

Let $s_{1}=t_{0}+\tau_{0}$ and $s_{2}=t_{0}+\tau$. For this case, the last formula can be proved in an analogous manner with the insignificant changes. It is clear that

$$
-\left[\int_{s_{1}}^{s_{2}} Y(s ; t) f_{y}[s] \dot{x}_{0}\left(s-\tau_{0}\right) d s\right] \delta \tau=o(t ; \delta \mu) .
$$

Consequently, for $R_{2}(t ; \delta \mu)$ we obtain

$$
\begin{gathered}
R_{2}(t ; \delta \mu)=-\left[Y\left(t_{0}+\tau_{0} ; t\right) \hat{f} \delta \tau+\int_{s_{1}}^{s_{2}} Y(s ; t) f_{y}[s] \dot{x}_{0}\left(s-\tau_{0}\right) d s\right] \delta \tau \\
+o(t ; \delta \mu)
\end{gathered}
$$


Similarly to the transformation of the expression $R_{1}(t ; \delta \mu)$, the expression $R_{3}(t ; \delta \mu)$ can be presented in the following form

$$
R_{3}(t ; \delta \mu)=\sum_{i=1}^{5} R_{3 i}(t ; \delta \mu)
$$

where

$$
\begin{gathered}
R_{31}(t ; \delta \mu)=\int_{s_{2}}^{t} Y(s ; t) \sigma_{1}(s ; \delta \mu) \Delta x(s) d s, \\
R_{32}(t ; \delta \mu)=\int_{s_{2}}^{t} Y(s ; t) \rho_{1}(s ; \delta \mu)\left[x_{0}(s-\tau)-x_{0}\left(s-\tau_{0}\right)+\Delta x(s-\tau)\right] d s, \\
R_{33}(t ; \delta \mu)=\int_{s_{2}}^{t} Y(s ; t) f_{y}[s]\left[x_{0}(s-\tau)-x_{0}\left(s-\tau_{0}\right)\right] d s \\
R_{34}(t ; \delta \mu)=\int_{s_{2}}^{t} Y(s ; t) f_{y}[s]\left[\Delta x(s-\tau)-\Delta x\left(s-\tau_{0}\right)\right] d s \\
R_{35}(t ; \delta \mu)=\int_{s_{2}}^{t} Y(s ; t) v_{1}(s ; \delta \mu) \delta u(s) d s .
\end{gathered}
$$

The function $x_{0}(s), s \in I$, is absolutely continuous, therefore, for each fixed Lebesgue point $s \in$ $\left(t_{0}+\tau_{0}, t_{1}\right)$ of the function $\dot{x}\left(\varsigma-\tau_{0}\right), \quad \varsigma \in\left(t_{0}+\tau_{0}, t_{1}\right)$, , we obtain

$$
\begin{aligned}
x_{0}(s-\tau)-x_{0}\left(s-\tau_{0}\right)= & \int_{s}^{s-\delta \tau} \dot{x}_{0}\left(\varsigma-\tau_{0}\right) d \varsigma=-\dot{x}_{0}\left(s-\tau_{0}\right) \delta \tau \\
& +\varrho(s ; \delta \tau)
\end{aligned}
$$

where

$$
\lim _{|\delta \tau| \rightarrow 0} \varrho(s ; \delta \tau) /|\delta \tau|=0 .
$$

From the boundedness of the function $\dot{x}_{0}(s), s \in I$ and (30), it follows that

$$
\left|x_{0}(s-\tau)-x_{0}\left(s-\tau_{0}\right)\right| \leq O(\delta \mu)
$$

and

$$
|\varrho(s ; \delta \tau)| /|\delta \tau| \leq \text { const }
$$

a.e. on $I$.

We note that for the compact sets $K_{1} \subset O$ and $U_{1} \subset U$, there exists a number $L>0$ such that

$$
\left|f\left(t, x_{1}, y_{1}, u_{1}\right)-f\left(t, x_{2}, y_{2}, u_{2}\right)\right| \leq L\left(\left|x_{1}-x_{2}\right|+\left|y_{1}-y_{2}\right|+\left|u_{1}-u_{2}\right|\right)
$$

for any $t \in I,\left(x_{i}, y_{i}\right) \in K_{1}^{2}, i=1,2$, and $\left(u_{1}, u_{2}\right) \in U_{1}^{2}$, (see [9], p. 29).

Furthermore,

$$
\begin{gathered}
\left|\Delta x(s-\tau)-\Delta x\left(s-\tau_{0}\right)\right| \leq\left|\int_{s-\tau_{0}}^{s-\tau}\right| \dot{\Delta x}(\varsigma)|d s| \leq\left|\int_{s-\tau_{0}}^{s-\tau}\right| a(s ; \delta \mu)|d s| \\
\leq L\left|\int_{s-\tau_{0}}^{s-\tau}\left[|\Delta x(s)|+\left|x_{0}(s-\tau)-x_{0}\left(s-\tau_{0}\right)\right|+|\Delta x(s-\tau)|+|\delta u(s)|\right] d s\right| \\
\leq L O(\delta \mu)|\delta \tau| \leq L O(\delta \mu)|\delta \mu|=o(\delta \mu)
\end{gathered}
$$

(see (18), (32)). According to (32), (34), for the expressions $R_{3 i}(t ; \delta \mu), i=1, \ldots, 5$, we have

$$
\left|R_{31}(t ; \delta \mu)\right| \leq\|Y\| O(\delta \mu) \sigma_{2}(\delta \mu) ; \quad\left|R_{32}(t ; \delta \mu)\right| \leq\|Y\| O(\delta \mu) \rho_{2}(\delta \mu) ;
$$




$$
\begin{gathered}
R_{33}(t ; \delta \mu)=-\left[\int_{s_{2}}^{t} Y(s ; t) f_{y}[s] \dot{x}_{0}\left(s-\tau_{0}\right) d s\right] \delta \tau+\varrho_{1}(t ; \delta \mu) \\
\left|R_{34}(t ; \delta \mu)\right| \leq o(\delta \mu) ; \quad\left|R_{35}(t ; \delta \mu)\right| \leq\|Y\| O(\delta \mu) v_{2}(\delta \mu),
\end{gathered}
$$

where

$$
\varrho_{1}(t ; \delta \tau)=\int_{s_{2}}^{t} Y(s ; t) f_{y}[s] \varrho(s ; \delta \tau) d s
$$

Obviously,

$$
\begin{gathered}
\left|\varrho_{1}(t ; \delta \tau)\right| /|\delta \mu| \leq\|Y\| \int_{t_{0}+\tau_{0}}^{t_{1}}\left|f_{y}[s]\right||\varrho(s ; \delta \tau)| /|\delta \mu| d s \\
\leq\|Y\| \int_{t_{0}+\tau_{0}}^{t_{1}}\left|f_{y}[s]\right||\varrho(s ; \delta \tau)| /|\delta \tau| d s=o(\delta \mu)
\end{gathered}
$$

(see (31) and (33)). Thus,

$$
R_{3}(t ; \delta \mu)=-\left[\int_{s_{2}}^{t} Y(s ; t) f_{y}[s] \dot{x}_{0}\left(s-\tau_{0}\right) d s\right] \delta \tau+o(t ; \delta \mu) .
$$

On the basis of (28), (29) and (35), we obtain

$$
\begin{gathered}
R(t ; \delta \mu)=-\left[Y\left(t_{0}+\tau_{0} ; t\right) \hat{f} \delta \tau+\int_{t_{0}}^{t} Y(s ; t) f_{y}[s] \dot{x}_{0}\left(s-\tau_{0}\right) d s\right] \delta \tau \\
+o(t ; \delta \mu)
\end{gathered}
$$

From (22), by virtue of (36), we obtain (9), where $\delta x(t ; \delta \mu)$ has the form (10).

\section{Conclusions}

The Formulas (9) and (10) allow one to find analytical relations between solutions of the initial and the perturbed equations, which is important to construct an approximate solution of the perturbed Equation (see (17)) and to obtain equations in "variations" (see (13) and (15)); Two ways of finding an approximate solution are given. Besides, these formulas can be used to prove the necessary optimality conditions for the delay optimization problem with the discontinuous initial condition. Future work will consider controlled equations with several delays in the phase coordinates and controls.

Author Contributions: The contribution of the authors in the performance of the work is evenly distributed. All authors have read and agreed to the published version of the manuscript.

Funding: This work was supported partly by the Shota Rustaveli National Science Foundation (Georgia), Grant No. Ph.D.-F-17-89.

Conflicts of Interest: There is no conflict of interest.

\section{References}

1. Arino, O.; Hbib, M.L.; Ait Dads, E. (Eds.) Delay Differential Equations and Applications; Nato Science Series II; Mathematics, Physics and Chemistry; Springer: Berlin, Germany, 2006; Volume 205, 581p.

2. Hale, J. Theory of Functional Differential Equations; Mir Publishers: Moscow, Russia, 1984; 421p. (In Russian)

3. Kolmanovski, V.; Myshkis, A. Introduction to the Theory and Applications of Functional Differential Equations; Kluwer Academic Publishers: Dordrecht, The Netherlands, 1999; 643p.

4. Ogustoreli, N.M. Time-Delay Control Systems; Academic Press: New York, NY, USA, 1966; 323p.

5. Gabasov, R.; Kirillova, F. The Qualitative Theory of Optimal Processes; Nauka: Moscow, Russian, 1971; 507p. (In Russian)

6. Gamkrelidze, R.G. Principles of Optimal Control Theory; Plenum Press: New York, NY, USA, 1978; 175p.

7. Kharatishvili, G.L.; Tadumadze, T.A. Formulas for the variation of a solution and optimal control problems for differential equations with retarded arguments. J. Math. Sci. (N. Y.) 2007, 140, 1-175. [CrossRef] 
8. Neustadt, L.W. Optimization: A Theory of Necessary Conditions; Princeton University Press: Princeton, NJ, USA, 1976; 424p.

9. Tadumadze, T. Variation formulas of solutions for functional differential equations with several constant delays and their applications in optimal control problems. Mem. Differ. Equ. Math. Phys. 2017, 70, 7-97.

10. Shavadze, T. Local variation formulas of solutions for nonlinear controlled functional differential equations with constant delays and the discontinuous initial condition. Georgian Math. J. 2020. [CrossRef]

11. Tadumadze, T.; Dvalishvili, P.; Shavadze, T. On the Representation of Solution of the Perturbed Controlled Differential Equation with Delay and Continuous Initial Condition. Appl. Comput. Math. 2019, 18, 305-315.

Publisher's Note: MDPI stays neutral with regard to jurisdictional claims in published maps and institutional affiliations.

(C) 2020 by the authors. Licensee MDPI, Basel, Switzerland. This article is an open access article distributed under the terms and conditions of the Creative Commons Attribution (CC BY) license (http://creativecommons.org/licenses/by/4.0/). 